Itinéraires Itinéraires

Littérature, textes, cultures

2011-1| 2011

Les Mémoires, une question de genre?

\title{
Françoise Lavocat (dir.), La Théorie littéraire des mondes possibles
}

Paris, CNRS Éditions, 2010, 326 p. ISBN : 978-2-271-06967-2.

\section{Xavier Garnier}

\section{OpenEdition}

\section{Journals}

Édition électronique

URL : http://journals.openedition.org/itineraires/1682

DOI : $10.4000 /$ itineraires. 1682

ISSN : 2427-920X

Éditeur

Pléiade

Édition imprimée

Date de publication : 1 avril 2011

Pagination : 171-173

ISBN : 978-2-296-13692-2

ISSN : 2100-1340

Référence électronique

Xavier Garnier, «Françoise Lavocat (dir.), La Théorie littéraire des mondes possibles », Itinéraires [En ligne], 2011-1 | 2011, mis en ligne le 01 avril 2011, consulté le 22 septembre 2020. URL : http:// journals.openedition.org/itineraires/1682 ; DOI : https://doi.org/10.4000/itineraires.1682

Ce document a été généré automatiquement le 22 septembre 2020.

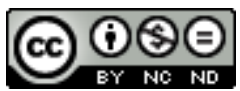

Itinéraires est mis à disposition selon les termes de la licence Creative Commons Attribution - Pas d'Utilisation Commerciale - Pas de Modification 4.0 International. 


\title{
Françoise Lavocat (dir.), La Théorie littéraire des mondes possibles
}

Paris, CNRS Éditions, 2010, 326 p. ISBN : 978-2-271-06967-2.

\author{
Xavier Garnier
}

\section{RÉFÉRENCE}

Françoise Lavocat (dir.), La Théorie littéraire des mondes possibles, Paris, CNRS Éditions, 2010, 326 p. ISBN : 978-2-271-06967-2.

1 L'ambition de cet ouvrage est double: faire un bilan de l'apport des théories des mondes possibles, nées chez les logiciens, aux approches littéraires des textes de fiction; interroger, par l'examen de textes de différentes époques, la genèse des procédures narratives qui ont rendu possible cette perception de mondes possibles, alternatifs ou parallèles. La catégorie des mondes possibles a l'avantage sur la catégorie plus large des mondes fictionnels de permettre de poser la question des rapports entre les fictions d'une part et entre la fiction et le monde réel. Anne Duprat définit en creux les mondes possibles en les distinguant des mondes fictionnels, qui «ne sont pas des hypothèses, parce qu'ils ne sont pas des ramifications d'un monde de référence qui serait le monde actuel» (p. 150). La dualité du virtuel et de l'actuel prend le relai de la dualité du fictif et du réel lorsqu'on cherche à saisir la spécificité des mondes possibles. La notion clé d'accessibilité, proposée par le logicien Kripke, et exploitée dans le volume par Philippe Monneret, rend bien compte de cette dynamique de ramification, qui suppose qu'un monde possible ne peut exister qu'en tant qu'il est accessible depuis un monde actuel de référence.

2 La première partie de l'ouvrage pose le problème du type de référentialité que l'on induit lorsqu'on fait intervenir la notion de «monde possible». Dans son article liminaire, Françoise Lavocat fait à la fois un récapitulatif des positions des pionniers de la théorie littéraire des mondes possibles et propose une typologie des genres de la fiction tenant compte de leurs différentes contributions. Le défi était de réconcilier 
dans une même typologie les conceptions des mondes possibles comme "mondes textualisés » (cf. Lucia Vaina d'une part et Uberto Eco d'autre part, qui évolue, avec la "fabula" vers une théorie de la lecture) et les conceptions des mondes possibles comme univers de référence seconds construits (Thomas Pavel, Marie-Laure Ryan, Lubomir Doležel). Marie Laure Ryan éclaire dans son article un clivage important, pour cette question de la référence, entre la position du logicien Saul Kripke pour qui le monde actuel est celui qui, dans la multiplicité des mondes possibles imaginables, échappe à l'imaginaire, pour s'actualiser dans la matière et devenir par là réel, et la position de David Lewis, pour qui les mondes actuels sont ceux qui embrayent sur des déictiques et donc varient en fonction du locuteur. Pour Lewis, il est possible d'actualiser des mondes virtuels tout en maintenant leur composante imaginaire. Lewis arrime ainsi les mondes actuels aux mondes textuels et ouvre la voie à la polyréférentialité. La référence de tout monde possible actualisé pourra alors être un autre monde possible, actualisé ailleurs, dans une dynamique textuelle très productive. C'est ainsi que Françoise Lavocat distinguera les fictions alternatives, les fictions autonomes et les transfictions en faisant jouer à chaque fois la possibilité pour la fiction de prendre comme référence d'autres fictions et démultipliant ainsi les niveaux de possibilités pour les mondes. Marie-Laure Ryan s'intéresse aux modélisations des mondes actuels en physique (notamment en mécanique quantique) pour tenter une description cosmologique des univers parallèles. Lubomir Doležel présente les procédures, les usages des récits contrefactuels du passé qui tirent leur valeur cognitive de cette concentration sur un moment de bifurcation possible entre ce qui a eu lieu et ce qui aurait pu avoir lieu. Richard Saint-Gelais exploite cette grande labilité des mondes possibles actuels pour analyser le potentiel théorique enveloppé dans les métalepses narratives, qui sont toujours des moments de saisissements susceptibles de déclencher une réflexion théorique.

3 La deuxième partie propose des «aperçus historiques" sur les modalités d'identification d'objets fictifs et la configuration de mondes fictifs à la Renaissance et au XVII ${ }^{e}$ siècle à la lumière de la théorie des mondes possibles. Marie-Luce Demonet pour la Renaissance, Anne Duprat pour l'univers baroque, Christine Noille-Clauzande pour la fiction au $\mathrm{XVII}^{\mathrm{e}}$ siècle examinent la mise en œuvre de paradoxes logiques embrayant une dynamique partant des objets fictifs (comme êtres de raison) aux mondes possibles, via les espaces imaginaires. Ce que la théorie des mondes possibles permet de voir, c'est la façon dont on a eu recours aux jeux paradoxaux pour créer un nouveau régime de la fiction au début de l'époque moderne. Partant des analyses de Kripke sur la nomination et le mode d'existence des entités fictionnelles, et de Lacan sur le nom de Joyce, l'article de Ruth Ronen voit dans l'activation du nom propre ce qui institue d'une façon immanente l'existence des entités fictionnelles dans leur singularité, au-delà du principe de vérité.

La troisième partie de l'ouvrage («Les mondes du texte: effets de lecture »), qui s'intéresse aux enjeux sociaux, éthiques voire politiques de l'usage des mondes possibles par le lecteur est autorisée par le parcours personnel de Thomas Pavel, tel qu'il le présente dans l'article qui clos l'ouvrage. Pavel explique que son travail sur les mondes possibles l'a amené progressivement à dépasser la logique formelle pour rendre compte de la fascination qu'exercent ces fictions sur le lectorat et le type de participation qu'il engage. Marielle Macé examine à partir de trois motifs narratifs (fictions théoriques, métalepses, micro-fictions) différentes façons dont le récit peut 
mettre en branle une « expérience de la fiction » qui est la condition d'une génération imaginaire des mondes possibles dont il est possible d'interroger la dimension cognitive. Sophie Rabau croise la théorie des mondes possibles et l'herméneutique classique sur la question de l'interférence entre les mondes esquissant ainsi une théorie des mondes impossibles résultants de la fusion de mondes, théorie qui pourrait nous éclairer sur ce que met en jeu l'acte de lecture dans notre rapport au monde. Marc Escola montre, à partir du cas particulier de «La belle au bois dormant» de Perrault, qu'un texte appelle des commentaires qui sont des mises en variation du monde fictionnel rendus possibles par le nombre nécessairement limité de propositions qui conditionnent ce monde. Le texte est un carrefour de possibles dont le commentateur a le loisir de trouver les points de bifurcation pour mettre en variation le monde, voire faire coexister des mondes compossibles. Les deux contributions de Philippe Monneret et d'Alexandre Gefen plaident pour un usage pragmatique de la théorie littéraire des mondes possibles en insistant, pour le premier, sur la capacité de ces mondes à intervenir au niveau du système de croyances et, pour le second, sur la démultiplication de l'expérience du réel rendue possible par cet élargissement des mondes.

\section{AUTEURS}

\section{XAVIER GARNIER}

Université Sorbonne-Nouvelle 